\title{
The Impact of Estrogen Depletion and Drug Treatment on Type I Collagen Microstructure
}

\author{
Meagan Cauble, ${ }^{1}$ Edward Rothman, ${ }^{4,5}$ Kathleen Welch, ${ }^{5}$ Ming Fang, ${ }^{1}$ Le T. Duong, ${ }^{6}$ Brenda L.
} Pennypacker, ${ }^{6}$ Bradford G. Orr, ${ }^{3}$ Mark M. Banaszak Holl. ${ }^{1,2}$

Departments of Chemistry, ${ }^{1}$ Biomedical Engineering, ${ }^{2}$ Physics, ${ }^{3}$ and Statistics ${ }^{4}$, and Center for Statistical Consultation and Research (CSCAR) $)^{5}$, University of Michigan, Ann Arbor, MI 48109. Bone Biology Group, ${ }^{6}$ Merck Research Laboratories, West Point, PA.

The reduction of estrogen level plays a significant role in Osteoporosis and Osteopenia, which affect over 73 million people. The impact of estrogen depletion on bone quality, and the effect of drugs used for treatment, have focused heavily on bone mineral density (BMD) as the primary experimental and clinical assay. However, BMD has demonstrated limitations for scientific understanding of the roles of estrogen in tissue as well as for clinical diagnosis and the prediction of fracture. ${ }^{1}$ The possible roles decreasing estrogen plays in reducing tissue quality remains an active area of research. An important avenue for study involves how protein structure and organization changes under conditions of estrogen depletion; however, this cannot be effectively measured by BMD so new structural metrics are needed. Atomic force microscopy (AFM) allows tissue level imaging of both micron scale tissue organization as well as measurement of individual Type 1 collagen fibril nanomorphology. ${ }^{2}$

In order to pursue these questions we have analyzed Type I collagen nanomorphology and microarchitecture for two studies employing overiectomized (OVX) rabbits as the model system for estrogen depletion. These models were previously employed for a comparison of the effects of alendronate (ALN) and cathepsin K inhibitors (CatKIs) on bone formation and resorption. ${ }^{3}$ One animal group included sham-operated animals, OVX animals, and OVX animals that were treated with either ALN or CatKi. The second animal group included sham-operated animals, OVX animals, and OVX animals given estrogen replacement therapy (ERT). These experiments provide two biologically independent tests of the changes in Type I collagen structure occurring under OVX estrogen depletion conditions. In addition, the impact of ALN, a bis(phosphonate) drug currently used in the clinic, CatKi, an example of an inhibitor that has just completed phase III clinical trials, and ERT on the protein structure was also explored. For all samples, AFM images of cortical femur bone were obtained.

A quantitative analysis of the AFM images of the micron level Type I collagen fibril organization indicates a significant decrease in fibrils appearing in ordered structures such as bundles and sheets (18\%), and a concomitant increase in fibrils appearing in disordered structures, upon OVX treatment of 6- and 9-month old rabbits. ALN and ERT drug treatment reduce the decrease of fibrils observed ordered structures (12\%) and CatKi provide an even greater degree of prevention resulting in only a 5\% decrease. These changes in the microscale level structure of the protein are mirrored by change in both structural bone properties, as indicated by average stiffness, and materials bone properties, as indicated by average strain energy. These studies point to the importance of gaining a further understanding of how changes in Type I collagen protein structure are related to estrogen-related disease for purposes of optimizing diagnosis, treatment, and the understanding of the impact on bone quality and function. 
A) Ordered Collagen Image

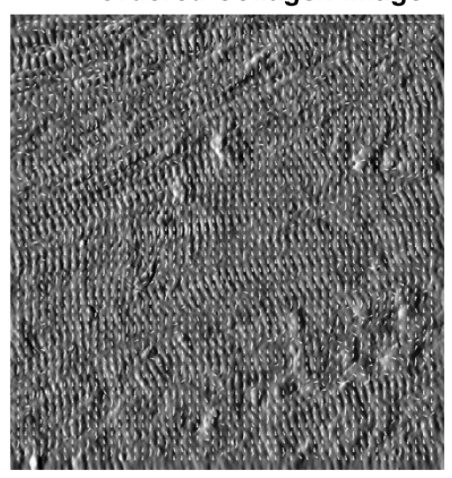

A) Disordered Collagen Image

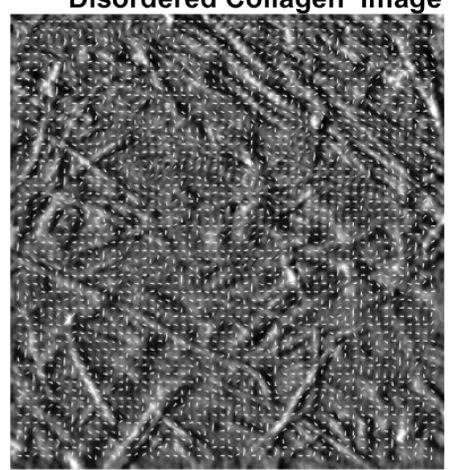

B)

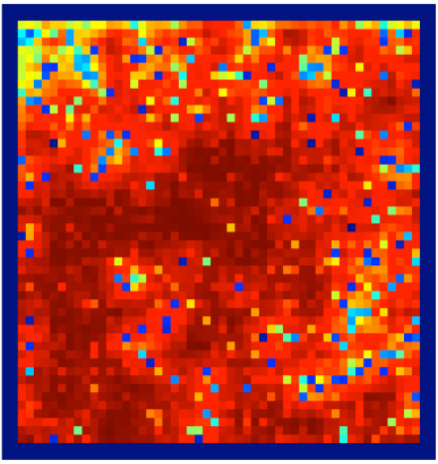

B)

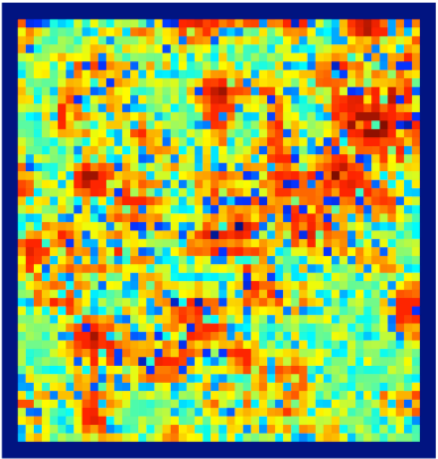

C)

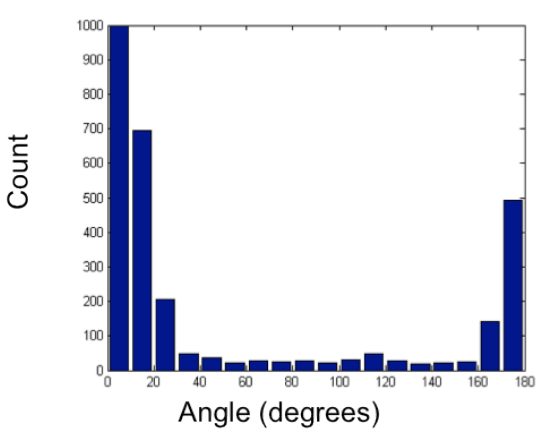

C)

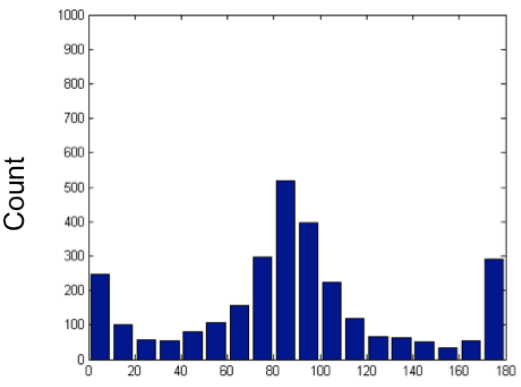

Angle (degrees)

Fig. 1 AFM images illustrating "ordered" and "disordered" regions of Type I collagen fibrils. A) images overlayed with vector map B) heat map indicating regions of local order C) angular distribution of vector overlay.

\section{References}

1. Burr, D.B. The contribution of the organic matrix to bone's material properties. Bone 31, 8-11 (2002). 2. Erickson, B., Fang, M., Wallace, J.M., Orr, B.G., Les, C.M. \& Banaszak Holl, M.M. Nanoscale structure of type I collagen fibrils: Quantitative measurement of D-spacing. Biotechnology Journal 8, 117-126 (2013).

3. Pennypacker, B.L., Duong, L.T., Cusick, T.E., Masarachia, P.J., Gentile, M.A., Gauthier, J.Y., Black,

W.C., Scott, B.B., Samadfam, R., Smith, S.Y. \& Kimmel, D.B. Cathepsin K Inhibitors Prevent Bone Loss in Estrogen-Deficient Rabbits. Journal of Bone and Mineral Research 26, 252-262 (2011).

4. The authors acknowledge funding from Merck, Inc for support of this work. 